\title{
A Novel MAC Algorithm for Energy Aware Wireless Sensor Networks
}

\author{
M. Premkumar \\ Assistant Professor/ECE \\ Sri Subramanya College of \\ Engineering and Technology \\ Palani, Tamil Nadu, India
}

\author{
M.Kathiravan \\ Assistant Professor/ECE \\ Dr. Nallini Institute of \\ Engineering and Technology \\ Dharapuram, Tamil Nadu, India
}

\author{
R. Thirukkumaran \\ M.E.-communication systems \\ Karpagam College of \\ Engineering \\ Coimbatore, Tamil Nadu, India
}

\begin{abstract}
Many Medium Access Control (MAC) protocols have been specifically designed for Wireless Sensor Networks (WSN) where energy awareness is an essential design issue. Sensor nodes sense environmental conditions, such as light, temperature, sound, or vibration etc., and transmit the sensed data to the sink node through multi-hop communication links. Energy awareness is one of the most important issues in WSNs. The radio transceiver is the most power consuming component in a sensor node. Transceiver power consumption is varying with different modes like transmit, receive, listen, and sleep. By using MAC protocol we can able to switch the radio interface into different modes. A new approach of an Energy Aware MAC (EA-MAC) algorithm is proposed in this paper. Also, we compare EA-MAC (proposed approach) with S-MAC (Sensor MAC) and ML-MAC (Multi-Layer MAC) based on Energy consumption, throughput and average endto-end delay. From this comparison study, we conclude that EA-MAC algorithm is better in the case of consumed less energy and sending more data than S-MAC and ML-MAC.
\end{abstract}

\section{General Terms}

Wireless sensor network, medium access control, energy efficiency, network simulation..

\section{Keywords}

Network lifetime, layer duration, Energy Aware MAC.

\section{INTRODUCTION}

A sensor is a small battery powered device. It senses the data from a node and transmits to another. Here the data's are transmitted over a radio frequency channel. A wireless sensor networks consist of large number of sensors deployed in an ad hoc manner. The sensors deployed according to the users applications. The WSN's is used in many applications such as military target tracking and surveillance $[1,2]$ natural disaster relief [3] biomedical health monitoring $[4,5]$ and hazardous environment exploration and seismic sensing [6]. In wireless sensor networks, the sensor battery is not possible to recharge because of harsh deployment. There is no secondary battery or backup in WSN's. So the usage of power in WSN's is very important. We have to utilize the power as much as possible to increase the network lifetime maximum. Normally the power loss is due to a crash between the data's, delay, overhearing, control packet overhead and idle listening [7]. Idle listen of sensor nodes are impact energy consumption. The effective mechanism to reduce power consumed by the wireless sensor networks is sleep-wake scheduling [8]. In this mechanism, the node is putting in to sleep mode when there is no data transmission. This reduces the power consumed by the wireless sensor networks. Many typical existing MAC protocols are designed to save power by placing the radio in the low-power sleep mode such as SMAC, TMAC, BMAC, wiseMAC and MLMAC.

The MAC protocols are basically divided into two types, based on the way how medium is accessed. These are synchronous (or Schedule) and asynchronous (or random access) MAC Protocols [9].

In synchronous MAC protocols, the channel is schedule for the nodes for some period of time. Because of scheduling this protocol have some demerits such as coordination to allocate and maintain the reservation slots, clock synchronization and lack of scalability. So the implementation is very difficult in WSN's, but it provides collision free data transmission. Therefore, synchronous MAC protocols reduce the energy consumption from most of the major sources of energy waste, i.e., idle listening, collision, and overhearing.

In Asynchronous MAC Protocols Nodes do not synchronize time and each sensor node contends for access to the radio channel. To reduce idle listening, protocols in this class add additional header information (i.e., the preamble) in the MAC frame, it allows nodes to check the channel periodically and sleep most of the time [9].It adds additional communication cost.

S-MAC [10] is a low power RTC-CTS based MAC protocol that makes use of loose synchronization between nodes to allow for duty cycling in sensor networks. In S-MAC, active period is of constant length. So Energy waste caused by idle listening is reduced by sleep schedules and also easy to implement. The protocol uses three techniques to achieve low power duty cycling: periodic sleep, virtual clustering, and adaptive listening. This also has some demerits because of fixed scheduling. If there is no traffic flow actually occurs, nodes stay wake needlessly long. This leads sensor nodes to energy waste. The node is active for long time when there is no data transmission. It will be waste of energy. By setting the node to sleep mode automatically while there is no data transmission, it will save the energy.

In TMAC [11] the nodes transmit all messages in bursts of variable length and sleep between bursts. The RTS-CTS-ACK schemes are used in TMAC for data transmission and synchronization is similar to S-MAC. TMAC improves on SMAC by assigning small wakeup period when it is IDLE. This will give better result under variable data transmission. Here the active period is dynamically variable. But it suffers from an early sleeping problem due to the asymmetric communication, but it overcomes the problem using FRTS (Future-Request-To-Send). 
Low Power Listening (LPL) [9] protocol does not try to fully synchronize the nodes. These protocols allow the receiver to sleep most of the time and only periodically sample the channel. Senders use long preambles to ensure that receiver stays wake to catch actual packet. Which provides low power for low traffic and do not incur overhead due to synchronization. The energy consumed on preamble transmission and reception after the receiver has woken up is wasted due to lack of information at the sender side about the wake-up schedule of the receiver, and thus the preamble length is chosen conservatively. Neighbor nodes other than the intended receiver will also be kept wake by the long preamble until the data packet transmission finishes, which is also wasteful since they are doing unneeded preamble overhearing. The target node has to wait for the full preamble before exchanging the data; this will increase latency at each hop.

The combination of CSMA and LPL techniques used in BMAC [12]. It is a carrier sense MAC protocol for WSN's. BMAC uses unsynchronized duty cycling and uses long preambles to wake up receivers. It uses the clear channel assessment (CCA) techniques to decide whether there is a packet arriving when node wakes up. While data transmission, timeout puts node back to sleep if no packet arrived. So idle Listening is reduced to a minimum. It has a better overall performance than S-MAC. The disadvantage BMAC is overhearing problem is not solved and it has lower duty cycle hence leads to higher average latency, higher cost to send, higher cost to overhear and hence more contention.

In wiseMAC [5], all nodes in a network sample the medium with a common period, but their relative schedule offsets are independent. If a node finds the medium busy after it wakes up and samples the medium, it continues to listen until it receives a data packet or the medium becomes idle again. The nodes learn and refresh their neighbor's sleep schedule during every data exchange as part of the Acknowledgment message. Every node keeps a table of the sleep schedules of its neighbors and decides own schedule accordingly. It performs better than S-MAC in various traffic load. The disadvantage of this wiseMAC is decentralized sleep-listen scheduling results in different sleep and wake-up times for each neighbor of a node. In broadcast-type communication packets are buffered for neighbors in sleep mode and delivered many times as each neighbor wakes up this causes higher latency and power consumption.

A multi-layer MAC (ML-MAC)[15] protocol is a technique to reduce node power consumption beyond that achieved by SMAC. It is also a self-organizing MAC protocol that does not require a central node to control the operation of the nodes. In ML-MAC time is divided into frames and each frame is divided into two periods: listen and sleep. The active period is sub-divided into $\mathrm{n}$ no of non-overlapping layers. Nodes are distributed among this set of layers where nodes in each layer follow a listen/sleep schedule that is skewed in time compared to the schedules of the other layers. A node in ML-MAC protocol wakes up only at its assigned layer. Therefore, ML-MAC requires a lesser amount of energy than S-MAC because the listen period of a node in ML-MAC is shorter than the listen period of the frame in S-MAC.

\section{PROPOSED METHOD}

EA-MAC is a contention based MAC protocol. It is a modified version of ML-MAC. In existing method half of the frame duration allotted for the sleep period and remaining allotted for listen period. This listen period further divided into $\mathrm{N}$ number of non overlapping Layers. In each frame duration only one layer is activated and the remaining time is allotted for sleep period. If the number of layers increased the retransmission of packets and delay will also be increased

Here the total network lifetime is divided into number of frames Nf. The frame duration is further divided into number of layers. Layer duration (TL) is calculated as total frame duration (TF) divided by number of layers. The period of each layer is fixed one. Fig.1 shows the layer architecture of EAMAC. By activating different layers in particular one frame duration we can change the listen and sleep periods adaptively.

Nodes within the coverage area of one sensor node is called neighbors. Selection of layers is based on number of neighbors for the particular sensor node and how much bandwidth is consumed by the neighbors. Common channel is shared among neighbor nodes. In our EA-MAC protocol all the nodes contend for the channel and eventually transmit without collisions.

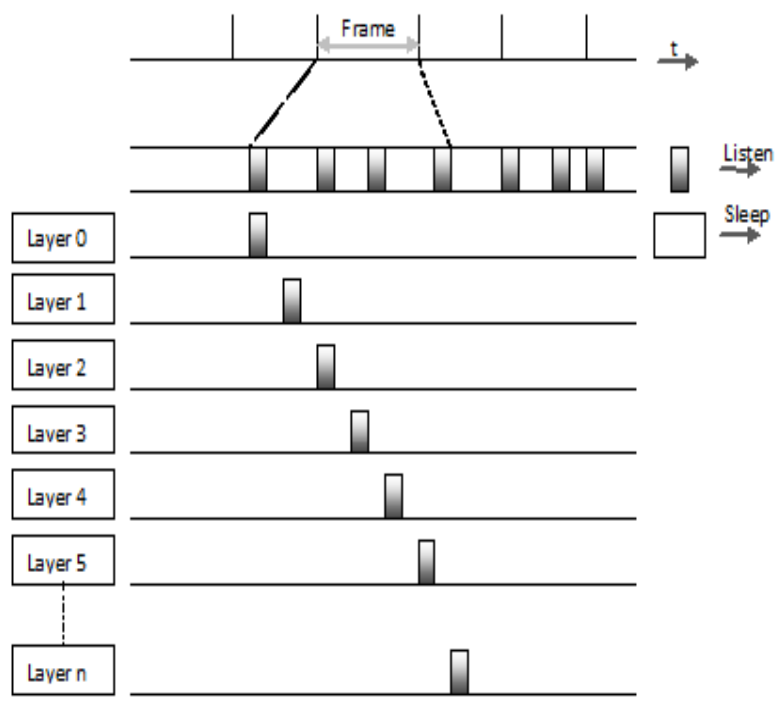

Fig 1: EA-MAC Architecture

The entire sensor node maintains the one hop neighbor information by periodically broadcasting a hello messages. Fig.2 shows Hello message it contains the information of Node Id, BW (Bandwidth consumed), Energy (Remaining energy), Packet Interval. Neighbor node information stored in neighbor table.

\begin{tabular}{|l|l|l|l|}
\hline Node ID & BW & Energy & Packet Interval \\
\hline
\end{tabular}

\section{Fig 2: Hello Message Structure}

If two nodes are trying to access the same channel at same time, collision will occur. BW consumed by the neighbors are increased less number of layers are activated.

Any sensor node wants to send data, first it checks the neighbor table if the BW consumed by the neighbors are greater than the threshold instead of contend the channel; node will buffer the data up to the next period. It avoids frequent retransmission of packets and collision. Layers are activated depends on the number of neighbor, packet size and data interval. 
Fig. 3 describes our proposed EA-MAC algorithm. Initially all the nodes activate only one layer randomly as listen period. Remaining layers will be in sleep state. Thus most of the energy conserved by all the nodes. If contention happens among neighbors retransmission will occur. This will increase unnecessary delay and bandwidth loss. To avoid this change the number of active layers with respect to bandwidth consumed by neighbors. Remaining bandwidth calculated as difference between maximum data rate to total neighbor consumed bandwidth. Number of active layers (NLA) calculated as remaining bandwidth (bw_r) divided by layer duration (TL). NLA should be less than total number of layers.

Based on NLA value different number of layers activated in consecutive frame duration. This will further reduce retransmission of packets and collision.

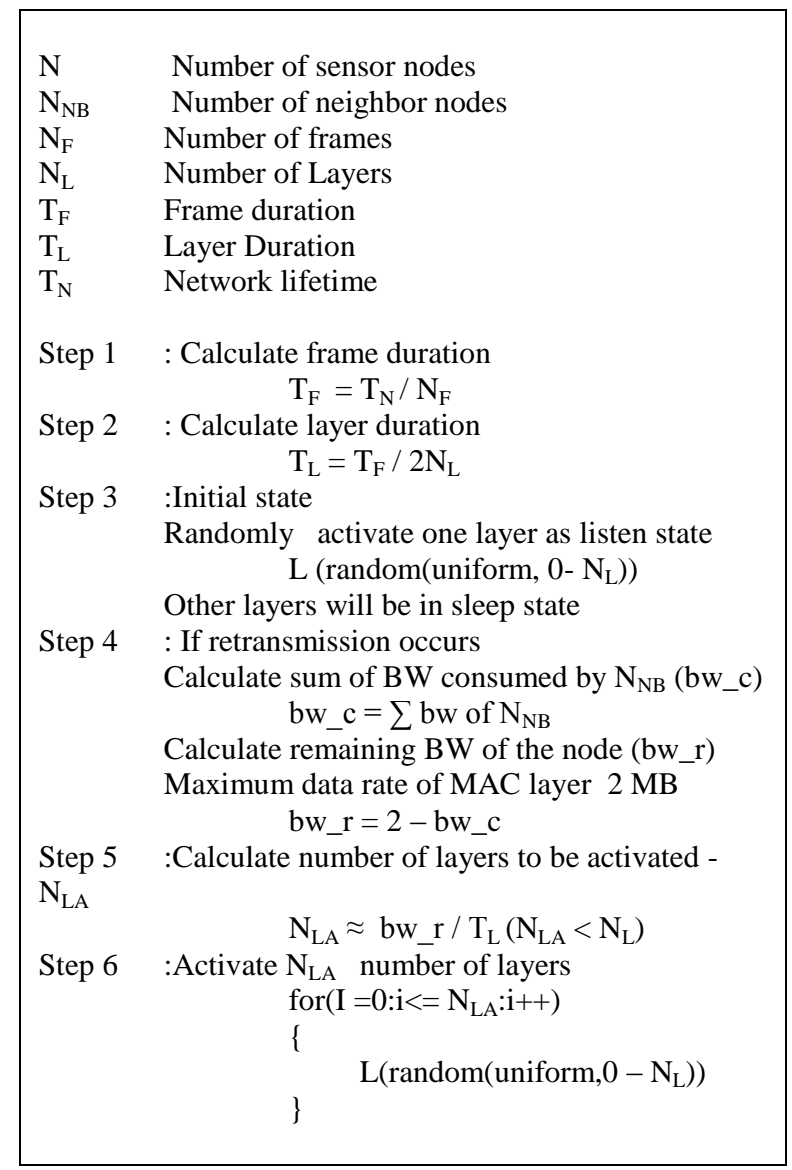

Fig 3: EA-MAC proposed algorithm

\section{PERFORMANCE ANALYSIS}

We evaluate EA-MAC protocol performance through NS$2[14]$ simulations. In particular we used NS-2.32 package as our tool to simulate the proposed protocol. NS-2 is an objectoriented discrete time event simulator written in $\mathrm{C}++$, with an OTcl interpreter and its modular design made it to be extensible.

Simulation parameters are listed in Table.1. We created a 100 sensor nodes and all are randomly placed within a $1000 \mathrm{~m} \mathrm{x}$ $1000 \mathrm{~m}$ area. Sensor node coverage area is defined as $100 \mathrm{~m}$. Each simulation is executed for 200 seconds. The Constant Bit Rate (CBR) application used between randomly selected source and destination pair. The size of the data packet is 38 bytes and the transmission rate varied from $0.1 \mathrm{~s}$ to $0.01 \mathrm{~s}$.

Table 1. Simulation parameters

\begin{tabular}{|l|l|l|}
\hline S. No & Parameter & Values \\
\hline 1 & Simulation area & $1000 \times 1000 \mathrm{~m}$ \\
\hline 2 & Node coverage area & $100 \mathrm{~m}$ \\
\hline 3 & Number of nodes & 100 \\
\hline 4 & Simulation time & $200 \mathrm{~s}$ \\
\hline 5 & Packet interval time & 0.01 to $0.1 \mathrm{~s}$ \\
\hline 6 & Average packet size & $38 \mathrm{Bytes}$ \\
\hline 7 & Number of layers & $1-10$ \\
\hline 8 & Initial Energy & $100 \mathrm{~J}$ \\
\hline 9 & Node transmitting power & $24.75 \mathrm{~mW}$ \\
\hline 10 & Node listening power & $13.5 \mathrm{~mW}$ \\
\hline 11 & Node sleeping power & $15 \mu \mathrm{W}$ \\
\hline
\end{tabular}

Average energy consumed per node is calculated as the summation of consumed energy of all nodes divided by number of nodes.

Packet end to end delay is calculated by subtracting the time at which packet is received by the destination from the time it was delivered from the source.

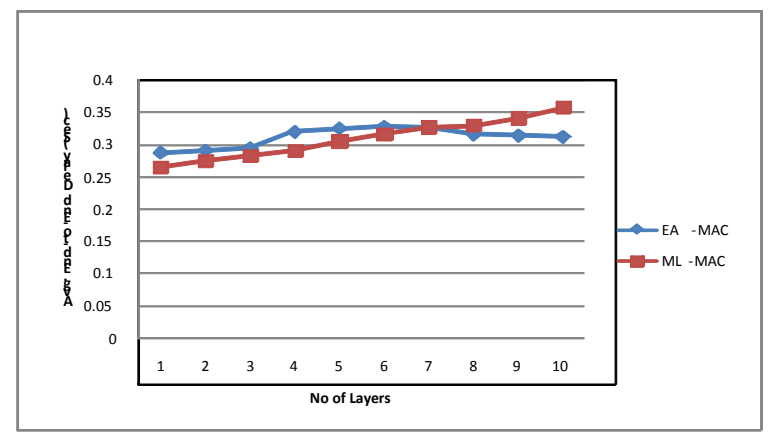

Fig 4: No of Layers Vs Avg. End-to-End Delay (sec)

In our proposed algorithm the number of layers activated in particular frame duration is varied with respect to the applied data traffic. The Fig. 6 shows our protocol consumed less energy compared with S-MAC and ML-MAC. In general, if the input interval decreases, data rate will be increased and the packet delivery ratio will be decreased.

We performed the simulation by varying the number of layer as well as packet interval parameters. While varying the number of layer parameter all other parameters are kept constant. Number of layer varied from $1-10$ and data packet interval is fixed as 0.02 seconds. 10 source and destination pairs are randomly selected. 


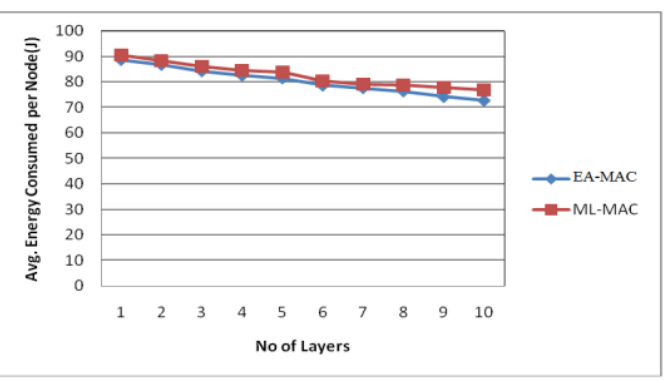

Fig 5: No of Layers Vs Avg. Energy Consumed per Node $(\mathbf{J})$

Fig.4 and Fig.5 shows our protocol performed well in terms of average energy consumed per node and average endto-end delay than S-MAC and ML-MAC.

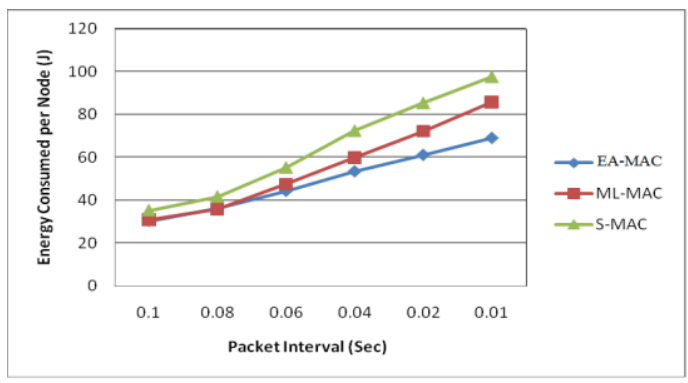

Fig 6: Packet Interval Vs Energy Consumed per Node (J)

\section{CONCLUSION}

Our simulation results show that EA-MAC protocol improves over S-MAC and ML-MAC in terms of energy consumption and average end-to-end delay. Network lifetime is also increased even high data rate applied. Our future work is to modify our EA-MAC protocol that can achieve both energy efficiency and bandwidth guarantee.

\section{REFERENCES}

[1] Balogh. G., Frampton. K., Kusy.B., Ledeczi. A., Maroti. M., Nadas. A., Pap. G., Sallai. J. and Simon. G. 2004 Sensor network-based countersniper system, Proceedings of the Second International Conference on Embedded Networked Sensor Systems (Sensys), Baltimore.

[2] Ghosal. D., Mukherjee. B. and Yick. J. 2005 Analysis of a Prediction-based Mobility Adaptive Tracking Algorithm, Proceedings of the IEEE Second International Conference on Broadband Networks.

[3] Castillo-Effen. M., Jordan. R., Quintela. D.H. and Westhoff. W. 2004 Moreno, Wireless sensor networks for flash-flood alerting, Proceedings of the Fifth IEEE International Caracas Conference on Devices, Circuits, and Systems, Dominican Republic.

[4] Alm. A., Gao. T., Greenspan. D., Juang. R.R., and Welsh. M. 2005. Vital signs monitoring and patient tracking over a wireless network, Proceedings of the 27th IEEE EMBS Annual International Conference.

[5] Clavel. A., Fulford-Jones. T.R.F Lorincz. K., Mainland. G., Malan. D., Moulton. S., Nawoj. A., Shnayder. V. and Welsh. M. 2004. Sensor networks for emergency response: challenges and opportunities, Pervasive Computing for First Response (Special Issue), IEEE Pervasive Computing.
[6] Johnson. J., Lees.v, Lorincz. K., Marcillo. O., Ruiz. M., Walsh. M. and Wener-Allen. G., 2006. Deploying a wireless sensor network on an active volcano, DataDriven Applications in Sensor Networks (Special Issue), IEEE Internet Computing.

[7] Estrin. D., Heidemann. J. and Ye. W. 2002. An energyefficient MAC protocol for wireless sensor networks. Proceedings of the 21st International Annual Joint Conference of the IEEE Computer and Communications Societies (INFOCOM 2002), vol. 3. p. 1567-76.

[8] Joohwan Kim, Ness. B., Prasun Sinha and Xiaojun Lin. 2010. Minimizing Delay and Maximizing Lifetime for Wireless Sensor Networks with Anycast. IEEE/ACM Transactions on Networking, VOL. 18, NO. 2.

[9] Roy.A. and Sarma.N. 2010.Energy Saving in MAC Layer of Wireless Sensor Networks: a Survey, National Workshop in Design and Analysis of Algorithm (NWDAA), Tezpur University, India.

[10] Deborah Estrin, John Heidemann, and Wei Ye. 2002. An energy-efficient mac protocol for wireless sensor networks. In Proceedings of the IEEE Infocom, pages $1567-1576$.

[11] Koen Langendoen and Tijs van Dam. 2003. An adaptive energy-efficient mac protocol for wireless sensor networks. In Proceedings of the First ACM SenSys Conference, pages 171-180.

[12] Culler. D., Hill. J. and Polastre. J. 2004. Versatile low power media access for wireless sensor networks. In The Second ACM Conference on Embedded Networked Sensor Systems (SenSys), pages 95-107.

[13] Decotignie. J. and El-Hoiydi. A. 2005. Low power downlink mac protocols for infrastructure wireless sensor networks. ACM Mobile Networks and Applications, 10(5):675-690.

[14] Network Simulator 2, available at http://www.isi.edu/nsnam/ns/.

\section{AUTHORS PROFILE}

R.Thhirukkumaran received the Master degree from Karpagam college of Engineering, Coimbatore and the Bachelor degree from Kongu College of Engineering, Erode, in 2012 and 1995. His research interest also includes the field of multimedia wireless networks: convergence, DSP, QoS and security.

M. Premkumar is an assistant professor at the Department of Electronics and Communication Engineering, Sri Subramaya College of Engineering and technology - Palani,Tamilnadu. He received the Master degree from Karpagam college of Engineering, Coimbatore and the Bachelor degree from Sri Subramaya College of Engineering and technology, Palani, in 2012 and 2009. His research interests include authentication in ad hoc and sensor networks, and security in peer to peer systems.

M. Kathiravan is an assistant professor at the Department of Electronics and Communication Engineering, Dr. Nallini Institute of Engineering and Technology-Dharapuram, Tamil Nadu. He received the Master degree (2012) and the Bachelor degree (2008) from Karpagam college of Engineering, Coimbatore. His research interests include specification, verification and testing of communication protocols; network security and distributed systems. 